\title{
Estado poblacional del pepino de mar (Clase Holothuroidea) en el Caribe de Guatemala
}

\author{
Population status of Sea Cucumber (Class Holothuroidea) \\ in the Caribbean of Guatemala \\ Cristopher Avalos-Castillo, José R. Ortíz-Aldana’ Francisco Polanco-Vásquez, Alerick Pacay \\ Instituto de Investigaciones Hidrobiológicas, Centro de Estudios del Mar y Acuicultura, \\ Universidad de San Carlos de Guatemala, Guatemala
}

*Autor al que se dirige la correspondencia: cristopheravalos@hotmail.es

Recibido: 27 de marzo 2017 / 1era. Revisión: 28 de febrero 2018 / 1era. Revisión: 11 de junio 2018 / Aceptado: 17 de junio 2018

\section{Resumen}

T os holoturoideos o pepinos de mar pertenecen al filo Echinodermata, e incluyen animales con cuerpo vermiforme Lalargado y blando que viven en los fondos de los mares de todo el mundo. Ecológicamente estos organismos son recicladores eficientes de sedimentos en el ambiente. En los últimos años han cobrado alto valor comercial en el mercado asiático, por lo que su pesquería se ha expandido hacia muchos países. En Guatemala se decretó una veda precautoria en el 2010 por cinco años, al terminar esta se extendió por dos años más, pero con el compromiso de obtener información científica durante dicho período. Con este fin, se realizó una evaluación poblacional del pepino de mar a través de transectos lineales de $60 \mathrm{~m}^{2}$, en siete puntos de la bahía de Amatique y la zona expuesta del mar Caribe de Guatemala, durante abril, junio y septiembre del 2016. Se evaluaron 6,300 $\mathrm{m}^{2}$, encontrando un total de 64 individuos de pepinos de mar. La riqueza de holotúridos está representada por siete especies, siendo las más abundantes Isostichopus badionotus (Selenka, 1867) y Holothuria mexicana (Ludwig, 1875). Los sitios con mayor abundancia fueron Faro Rojo y King Fish $(p=.004)$. En base a los resultados de esta evaluación se puede determinar que la explotación comercial de los pepinos de mar en el Caribe guatemalteco no es viable, y que para aprovechar el valor comercial de estas especies se podría impulsar la acuicultura y comercializarlo a través de un sistema eficiente de trazabilidad.

Palabras claves: Isostichopus badionotus, Holothuria mexicana, riqueza, abundancia, pesquería

\section{Abstract}

$\mathrm{H}$ olothurians or sea cucumbers belong to the filum Equinodermata, which includes animals with long soft vermiform bodies that live on the ocean bottom of the entire world. Ecologically these organisms are efficient recyclers of sediments in the environment. In recent years, they have gained high commercial value in the Asian market, so their fishery has expanded to many countries. In Guatemala, a precautionary ban was decreed in 2010 for five years, when it ended it was extended for two more years, but with the commitment to obtain scientific information during that period. For this purpose, a population assessment of sea cucumber was carried out through linear transects of $60 \mathrm{~m}^{2}$, in seven points of Amatique Bay and the exposed zone of the Caribbean Sea of Guatemala, during April, June and September of 2016. $6300 \mathrm{~m}^{2}$ were evaluated, founding 64 organisms. The holothurians richness is represented by seven species, being the most abundant Isostichopus badionotus (Selenka, 1867) and Holothuria mexicana (Ludwig, 1875). The most abundant sites were Faro Rojo and King Fish $(\mathrm{p}=.004)$. Based on the results of this evaluation, it can be determined that the commercial exploitation of sea cucumbers in the guatemalan Caribbean is not viable, and that to take advantage of the commercial value of these species, aquaculture could be promoted and marketed through an efficient traceability system. 


\section{Introducción}

Los holotúridos (Echinodermata) son organismos que se alimentan de materia orgánica, pudiendo remover grandes cantidades del fondo marino, siendo importantes en la mezcla de sedimentos y el reciclaje de materia orgánica en los ecosistemas. Por lo que han sido utilizados como bioindicadores de contaminación (Quintanal-López, Burgos-Suarez, \& Lagunés-Vega, 2013; Ruíz, Ibáñez, \& Cáceres, 2007; Yingst, 1982). La mayoría de los pepinos de mar son dioicos, pero se han contabilizado algunas especies hermafroditas (Borrero-Pérez, Benavides-Serrato, \& Díaz-Sánchez, 2012; Fajardo-León, Suárez-Higuera, Valle-Manríquez, \& Hernández-López, 2008). Son organismos de tallas pequeñas de longitud total $(60 \mathrm{a} 160 \mathrm{~mm})$, tienen una movilidad limitada, madurez tardía, poseen una reproducción densodependiente y poseen bajos índices de reclutamiento, lo que los hace particularmente susceptibles a la sobreexplotación (Bordbar, Anwar, \& Saari, 2011; Rodríguez-Gil, Reyes-Sosa, Alpízar-Carrillo, \& Tello-Cetina, 2007).

El desarrollo de la pesquería de pepinos de mar a nivel mundial se ha dado principalmente por dos factores. El primero de ellos es por el incremento de la demanda internacional y aumento del precio en el mercado asiático, cotizándose entre 85 y 120 US\$ el kilo de pepino deshidratado (Salgado-Rogel et al., 2009; Tuz-Sulub \& Aguilar-Perrera, 2011). El otro factor que ha contribuido es la reducción de los stocks tradicionales de pesca, principalmente de escama y otras especies como abulón y erizos de mar, que ha hecho que se busquen nuevas especies que suplan las actividades pesqueras y que esto provea una alternativa de ingresos económicos (López-Rocha, 2012; Salgado-Rogel et al., 2009). Actualmente esta pesquería contribuye sustancialmente al desarrollo económico y social de las regiones costeras de muchos países como México, Ecuador y Estados Unidos (Chen, Feindel, Kirshenbaum, Nutting, \& Mercer, 2007; Tuz-Sulub \& Aguilar-Perrera, 2011).

Uno de los aspectos básicos para lograr un manejo efectivo en el inicio de una explotación pesquera es conocer la biología, ecología, distribución, abundancia y la manera en que la población se ve afectada por la presión de pesca (López-Rocha, 2012; Uthicke \& Benzie, 2011). Estos factores no fueron tomados en cuenta al abrir la pesquería de pepino de mar en ciertas regiones, lo que propició beneficios efímeros a los pescadores, provocando que en poco tiempo el recurso se reportara en estado de sobreexplotación; Estas experiencias fueron observadas en Baja California
(Consultores Acuícolas y Pesqueros S.C., 2007; Fajardo-León, Suárez-Higuera, del Valle-Manríquez, \& Hernández-López, 2008), Campeche (Espinoza-Tenorio, Pech, Ramos, \& Peña-Puch, 2012), y Yucatán en México (López-Rocha, 2012); Costa Rica (Cortés, 1997); Islas Galápagos en Ecuador (De Miras, Echeverría, \& Carraza-Barona, 1996; Toral, Martínez, Hearn, \& Vega, 2003); y en Australia (Uthicke \& Benzie, 2011).

En los países donde se realiza la pesca comercial y se ha llevado a una sobreexplotación de holotúridos, se han realizado esfuerzos en implementar medidas de ordenación pesquera de la especie. En Yucatán, México, en el 2007, se estableció como medida de ordenación, proponer cuotas de $2 \%$ de extracción sobre la abundancia estimada para dicho año (Poot-Salazar, Ardisson, Poot-Salazar, Poot-Salazar, \& Caro-Méndez, 2014). A pesar de ello en muchos países prevalece la pesca ilegal por lo que el recurso se encuentra en sobreexplotación (Consultores Acuícolas y Pesqueros S.C., 2007). Por esta razón, durante el 2014, se desarrolló un taller de la Convención sobre el Comercio Internacional de Especies Amenazadas de Fauna y Flora Silvestres (CITES por sus siglas en inglés) para la protección de pepino de mar en Malasia, donde se establecieron siete medidas de manejo para estas especies: talla mínima de captura (se reconoce que esta medida es difícil de manejar por los cambios en tamaño y forma de los pepinos de mar), declaración de áreas de no pesca donde se establezcan comunidades de pepinos de mar, control de la captura total permitida, restricción de aparejos de pesca, rotación de las áreas de pesca, vedas temporales y limitación al esfuerzo de pesca al controlar la cantidad de pescadores que pueden hacer uso del recurso (Bruckner, 2006).

En Guatemala, durante los últimos años de la década de los 2000, se inició la pesca de pepino de mar en la zona del Caribe del país, pero no existen datos sobre especies, capturas o exportaciones registradas durante ese periodo. El Consejo Nacional de Áreas Protegidas (Conap), por conocer el estado de la zona solicitó a la Dirección de Normatividad de la Pesca y Acuicultura (Dipesca), el establecimiento de una veda para las especies de pepino de mar, la cual fue realizada y entró en vigor el 1 de julio del 2010 (Ministerio de Agricultura, Ganadería y Alimentación [Maga], 2010). Al vencer el periodo de veda, surgió la presión social para que se abriera la pesquería, pero Dipesca aún no poseía datos de las especies, por lo que prorrogo la veda por dos años, con el deber de realizar investigación sobre ellas (Maga, 2015). 
A raíz de esta necesidad se investigó el estado de la población de pepino de mar, que contribuirá a la ordenación del recurso pesquero del país, utilizando metodologías para la evaluación de pepinos de mar en México (Consultores Acuícolas y Pesqueros, S.C., 2008). El principal objetivo del proyecto es contribuir con información científica a la dirección nacional de pesca para el manejo del recurso pepino de mar en el Caribe de Guatemala, con datos de abundancia y riqueza de especies.

\section{Materiales y métodos}

\section{Descripción del sitio}

El litoral Caribe de Guatemala está representado por una bahía formada por materiales aluviales; sus costas tienen origen sedimentario, presentando alta intensidad en la dinámica de sus playas y con litorales en permanente transformación (Fundación Mario Dary Rivera, Consejo Nacional de Áreas Protegidas, \& The Nature Conservancy, 2006).

La bahía de Amatique cuenta con dos áreas marinas protegidas: Áreas de Usos Múltiples Río Sarstún (AUMRS), y el Refugio de Vida Silvestre Punta de Manabique (RVSPM). Las cuales cuentan con ecosistemas de pastos marinos, suelos arenosos y arrecifes de coral. Sus aguas costeras son turbias y con alto contenido de sedimentos, lo cual no permite el crecimiento adecuado de las poblaciones de coral, sin embargo, se reporta presencia de arrecifes en el área, aunque su estado de salud está categorizado como "pobre". Lo cual significa que tiene poca abundancia de peces, alta cobertura de macroalgas y baja cobertura de corales (Healthy Reefs Initiative, 2015).

Guatemala tiene limitado desarrollo de arrecifes coralinos en la costa Caribe, los bancos carbonatados mejor conocidos son los ubicados en Punta de Manabique, los cuales están dominados por especies de corales resistentes a los sedimentos como la Siderastrea siderea (Ellis \& Solander, 1768) y con aisladas comunidades de coral y pequeños parches de arrecife en el Golfo de Honduras (Fonseca \& Arrivillaga, 2003; McField et al., s.f.).

\section{Muestreo}

El muestreo se llevó a cabo en siete puntos, cuatro dentro de la bahía de Amatique y tres en la parte expuesta del Caribe de Guatemala (Figura 1). Se realiza- ron tres visitas, en los meses de abril, junio y septiembre del 2016, realizando en cada punto, cinco transectos lineales de $30 \mathrm{~m}$ de largo por $2 \mathrm{~m}$ de ancho. Las profundidades variaron de 10 a $18 \mathrm{~m}$ de profundidad, y se realizó a través de buceos SCUBA (Consultores Acuícolas y Pesqueros S.C., 2008). Se realizaron un total de 105 transectos durante la investigación, cubriendo un área de $300 \mathrm{~m}^{2}$ por muestreo en cada sitio, con 6,300 $\mathrm{m}^{2}$ en todo el estudio. Al conocer el área muestreada se pudo dividir el número de individuos en cada muestreo por el área para calcular la densidad relativa. La identificación de los organismos se realizó a distintos niveles taxonómicos, con ayuda de guías especializadas (Borrero-Pérez, Benavides-Serrato, \& Díaz-Sánchez, 2012; Herrero-Pérezrul, 2015). El estudio se llevó a cabo en ecosistemas de arrecife donde existe predominancia de corales de los géneros Agaricia y Porites, pastos marinos compuestos principalmente por las especies Thalassia testudinum (Banks \& Sol. Ex K.D. Koenig) y Syringodium filiforme (Kütz), y parches de arena.

Los holotúridos observados fueron fotografiados y se recolectó un organismo por cada especie para su identificación taxonómica la cual fue realizada únicamente a través de sus características morfológicas. Los especímenes fueron depositados en la colección de referencia del Centro de Estudios del Mar y Acuicultura, bajo los números de referencia 492 al 496.

\section{Análisis estadístico}

Se realizaron análisis de varianza no paramétricos Kruskal-Wallis (1952) y Friedman-Dunn (1937) (InfoStat, 2008), los cuales fueron utilizados para evidenciar si existían diferencias significativas en cuanto a la abundancia de holotúridos en los diferentes sitios y meses de muestreo.

\section{Resultados}

A lo largo del período de investigación se registraron 64 individuos, de los cuales se pudo identificar a cinco especies: Actinopyga agassizi (Selenka, 1867), Astichopus multifidus (Sluiter, 1910), Holothuria (Halodeima) mexicana (Ludwing, 1875), Holothuria (Thymiosycia) thomasi (Pawson \& Caycedo, 1980) e Isostichopus badionotus (Selenka, 1867). Un grupo de individuos únicamente pudo identificarse a nivel de familia, correspondiendo a la familia Stichopodidae (Haeckel, 1896) (Figura 2). Los tres grupos más 


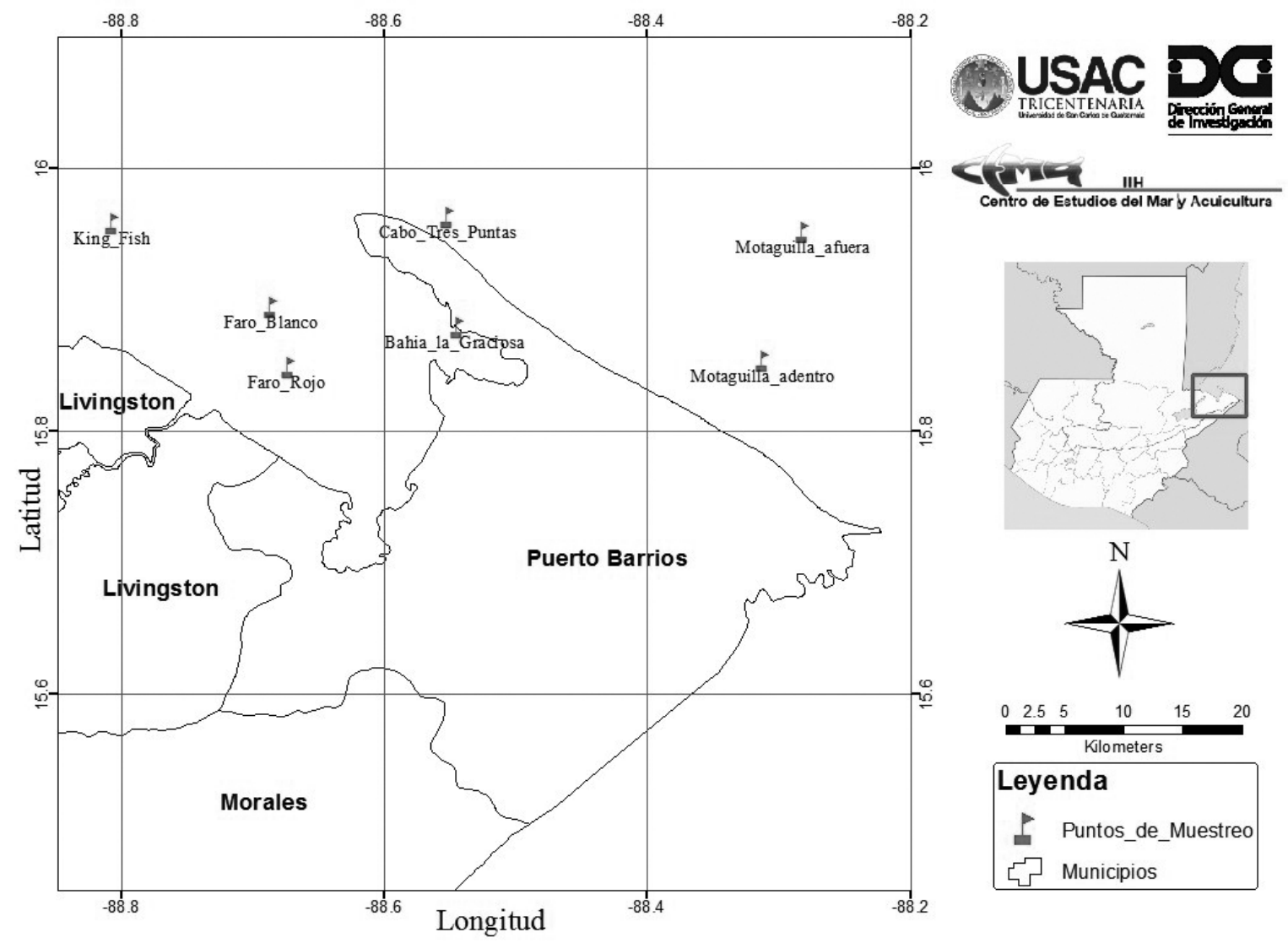

Figura 1. Puntos de muestreo en el Caribe de Guatemala

abundantes fueron: I. badionotus (29 individuos), los organismos de la familia Stichopodidae (16 individuos) y H. mexicana (15 individuos) (Tabla 1).

El sitio de muestreo que presentó mayor riqueza de especies fue Faro Rojo, seguido de King Fish y luego Motagüilla adentro y Cabo Tres Puntas. Faro Blanco y bahía La Graciosa fueron los puntos donde no se reportó la presencia de holotúridos (Tabla 2). En total se encontraron 64 pepinos de mar dentro y fuera de la bahía de Amatique, distribuidos en 24 individuos en abril, 12 en junio y 31 en septiembre (Tabla 3 ).

Mediante la prueba de Kruskal-Wallis se determinó que no hubo diferencia significativa $(p>.05)$, con respecto a la abundancia entre los sitios de muestreo. Sin embargo, utilizando la prueba de Friedman-Dunn se determinó diferencia significativa entre la abundancia de los puntos de Faro Rojo y King Fish con respecto a los otros puntos $(p=.004)$. También con la prueba de Friedman-Dunn se determinó similitud entre los meses de muestreo de abril y junio, así como entre los meses de junio y septiembre $(p=.043)$.

El punto que presentó mayor densidad (número de ind $/ \mathrm{m}^{2}$ ) fue Faro Rojo con una densidad promedio (desviación estándar) de 0.058 (0.024) ind $/ \mathrm{m}^{2}$, Kingfish con 0.011 (0.011) ind $/ \mathrm{m}^{2}$, seguido por Cabo Tres Puntas y Motagüilla adentro con $0.002(0.004) \mathrm{ind} / \mathrm{m}^{2}$ cada uno. El mes en el que se reportó la mayor densidad de pepinos de mar en la bahía de Amatique fue septiembre $\left(0.015(0.030) \mathrm{ind} / \mathrm{m}^{2}\right)$, seguido por abril $(0.011(0.020)$ ind $\left./ \mathrm{m}^{2}\right)$ y junio $\left(0.006(0.014) \mathrm{ind} / \mathrm{m}^{2}\right)$ (Tabla 4$)$.

\section{Discusión}

Los géneros de pepino de mar encontrados en las aguas del Caribe de Guatemala se encuentran entre el listado de especies de importancia comercial a nivel mundial (Toral-Granda, 2008), siendo estos los 


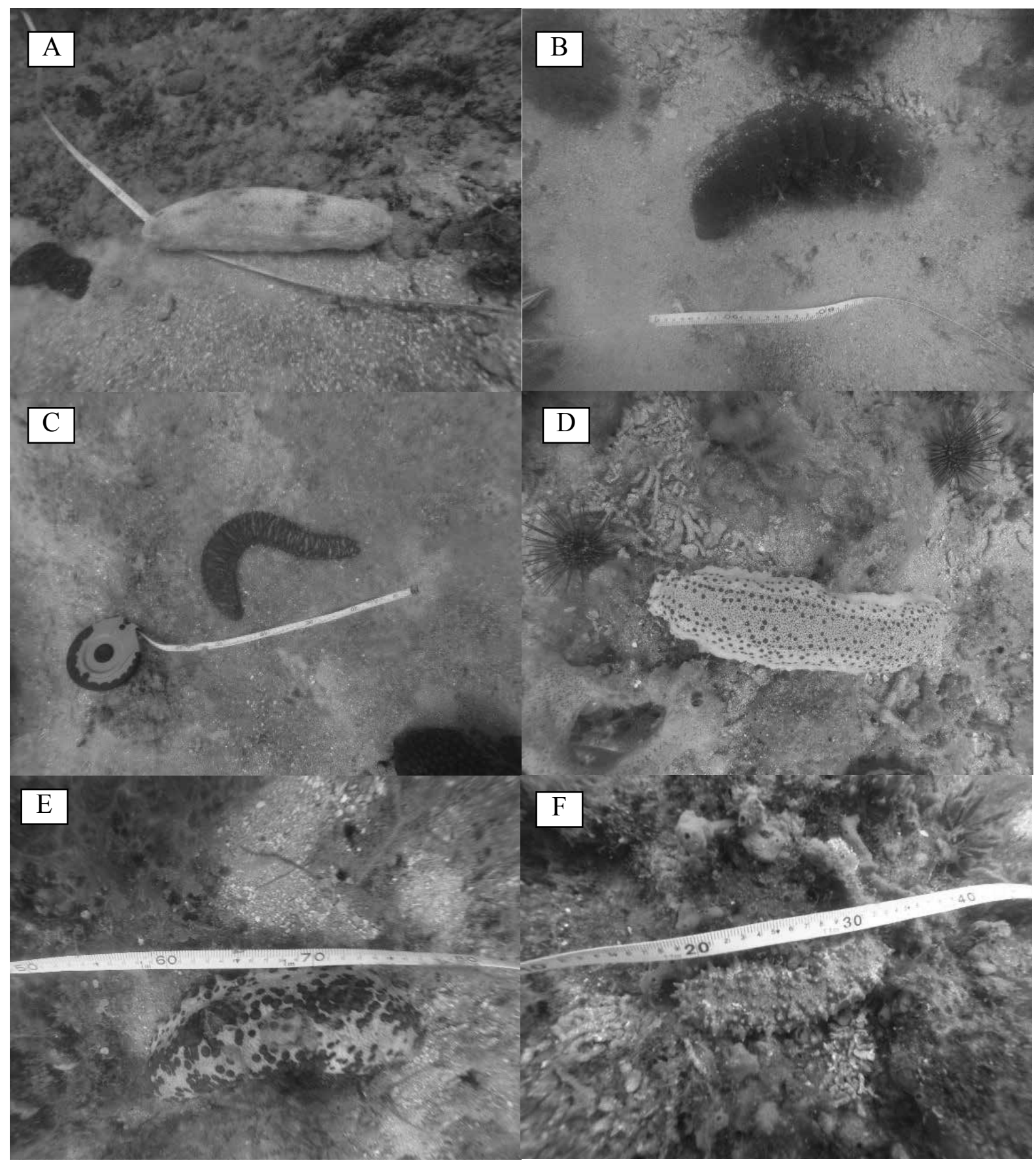

Figura 2. Especies de pepinos de mar identificados en el Caribe de Guatemala. (A) Astichopus multifidus; (B) Holothuria (Halodeima) mexicana; (C) Holothuria (Thymiosycia) thomasi; (D) y (E) variaciones de Isostichopus badionotus; (F) Stichopodidae sin determinar. 
Tabla 1

Especies de pepino de mar presentes y número de organismos observados en la bahía de Amatique, Izabal, Guatemala

\begin{tabular}{clc}
\hline Familia & \multicolumn{1}{c}{ Especie } & No. de individuos \\
\hline Holothuridae & Actinopyga agassizi (Selenka, 1867) & 2 \\
& Holothuria (Halodeima) mexicana (Ludwig, 1875) & 15 \\
& Holothuria (Thymiosycia) thomasi (Pawson \& Caycedo, 1980) & 1 \\
Stichopodidae & Astichopus multifidus (Sluiter, 1910) & 1 \\
& Isostichopus badionotus (Selenka, 1867) & 29 \\
& Stichopodidae & 16 \\
\hline
\end{tabular}

Tabla 2

Riqueza de especies por sitio de muestreo en bahía de Amatique, Izabal, Guatemala

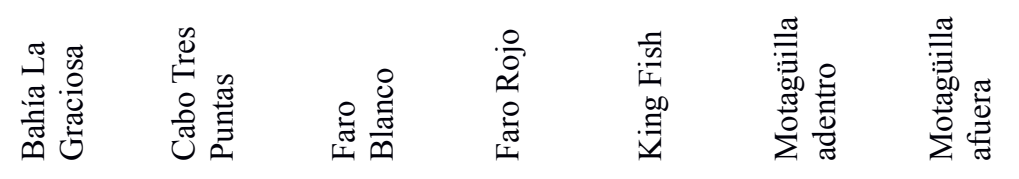

\begin{tabular}{|c|c|c|c|c|c|c|c|}
\hline \multirow{2}{*}{$\begin{array}{l}\text { Actinopyga agassizi } \\
\text { Holothuria mexicana }\end{array}$} & \multicolumn{7}{|c|}{$\mathrm{X}$} \\
\hline & & $\mathrm{X}$ & & & $X$ & $\mathrm{X}$ & $\mathrm{X}$ \\
\hline Holothuria thomasi & & $X$ & & & & & \\
\hline Astichopus multifidus & & & & & & $\mathrm{X}$ & \\
\hline Isostichopus badionotus & & & & $\mathrm{X}$ & & & \\
\hline Stichopodidae & & & & $\mathrm{X}$ & & & \\
\hline Total & 0 & 2 & 0 & 4 & 1 & 2 & 1 \\
\hline
\end{tabular}

Tabla 3

Número de pepinos de mar en el Caribe de Guatemala

\begin{tabular}{lcccc}
\hline Sitio & Abril & Junio & Septiembre & Total \\
\hline King Fish & 7 & 1 & 2 & 10 \\
Faro Blanco & 0 & 0 & 0 & 0 \\
Faro Rojo & 14 & 11 & 22 & 47 \\
Bahía La Graciosa & 0 & 0 & 0 & 0 \\
Cabo Tres Puntas & 0 & 0 & 2 & 2 \\
Motagüilla adentro & 0 & 0 & 2 & 2 \\
Motagüilla afuera & 1 & 0 & 0 & 1 \\
\hline Total & 22 & 12 & 30 & 64 \\
\hline
\end{tabular}


Tabla 4

Densidad de pepinos de mar en el Caribe de Guatemala

\begin{tabular}{lllll}
\hline Sitio & Abril $\left(\mathrm{ind} / \mathrm{m}^{2}\right)$ & Junio $\left(\mathrm{ind} / \mathrm{m}^{2}\right)$ & Septiembre $\left(\mathrm{ind} / \mathrm{m}^{2}\right)$ & Densidad promedio \\
\hline King Fish & 0.023 & 0.003 & 0.007 & $0.011(0.011)$ \\
Faro Blanco & - & - & - & - \\
Faro Rojo & 0.050 & 0.037 & 0.083 & $0.058(0.024)$ \\
Bahía La Graciosa & - & - & - & - \\
Cabo Tres Puntas & - & - & 0.007 & $0.002(0.004)$ \\
Motagüilla adentro & - & - & 0.007 & $0.002(0.004)$ \\
Motagüilla afuera & 0.003 & - & - & $0.001(0.002)$ \\
\hline Total & 0.011 & 0.006 & 0.015 & $0.011(0.005)$ \\
\hline
\end{tabular}

géneros Isostichopus, Holothuria, y Actinopyga. Los cuales se comercializan en Latinoamérica y el Caribe (Toral-Granda, 2008).

El único registro de especies de holotúridos en Guatemala fue publicado por Alvarado (2011), en el cual se indica que la diversidad de pepinos de mar está representada por cuatro especies: Eostichopus arnesoni (Cutess \& Miller, 1982), Actinopyga agassizi (Selenka, 1867), Holothuria (Halodeima) mexicana (Ludwing, 1875), y Holothuria (Thymiosycia) arenícola (Semper, 1868). De esta manera, las especies: A. multifidus, I. badionotus y H. (Thymiosycia) thomasi representan primeros registros para el Caribe de Guatemala.

Las especies I. badionotus, $H$. mexicana, A. agassizi, y $H$. thomasi actualmente han sido registradas en los tres países con los que se comparte el Sistema Arrecifal Mesoamericano, México, Belice y Honduras; mientras que la especie $A$. multifidus, solo ha sido registrado en México (Alvarado, 2011). De estas especies, H. mexicana, I. badionotus y A. multifidus son las que poseen mayor valor comercial, capturadas en diversas áreas del mar Caribe, como en Bocas del Toro, Panamá; Quintana Roo y Yucatán, México y, Colombia (Borrero-Pérez et al., 2012; De la Fuente-Betancourt, Guzmán \& Guevara, 2002; Navarrete, Sosa-Cordero, \& Herrero-Perezrul, 2001; ).

De los puntos de muestreo evaluados, solo uno de ellos presenta densidades de pepino de mar similares a los que se reporta en áreas donde existe actividad pesquera de extracción de pepino de mar. Este lugar es Faro Rojo, ubicado cerca del centro de la bahía de Amatique, donde se encontraron densidades entre 0.03 y 0.08 ind/ $\mathrm{m}^{2}$. Estos entran en el rango presentado en Yucatán para 2011 que fue de 0.04 pepinos de mar in $/ \mathrm{m}^{2}$ (Poot-Sala- zar et al., 2014). En Bocas del Toro, las densidades de pepinos de mar explotadas se encuentran en 0.016 ind/ $\mathrm{m}^{2}$, estimando poblaciones de H. mexicana de más de 7 millones de individuos en esa región, sin embargo estas densidades indican niveles críticos de sobrepesca en el área (Guzmán \& Guevara, 2002).

La densidad y diversidad de pepinos de mar en los transectos de sustrato coralino y algas fue mucho mayor que la que presentaron aquellos en donde había arena y pasto. Principalmente debido a que estos organismos viven, por lo general, en estructuras rocosas o coralinas que brindan refugio durante el día y pueden explorar en la noche debido a sus hábitos nocturnos (Ruppert \& Barnes, 1996). Al encontrarse bajas densidades de pepino de mar en el mar territorial del Caribe guatemalteco no se recomienda establecer una pesquería dirigida hacia esta especie.

Con base en los resultados obtenidos, durante la socialización de resultados con Dipesca a inicios de 2017, se recomendó continuar con la veda de pepinos de mar en el Caribe de Guatemala ya que la abundancia encontrada en aguas guatemaltecas no era suficiente para soportar la presión pesquera. Dipesca, utilizando esta recomendación y otros estudios prorrogó la veda de pepino de mar por cinco años más según Acuerdo Ministerial 101-2017, publicado en el Diario de Centroamérica el 5 de junio de 2017. Como apoyo a la veda, el departamento de pesca nacional podría generar acuerdos para controlar el comercio ilegal a través de controles en el comercio, especialmente en las exportaciones en aduanas, así como poder adaptar la tecnología necesaria para poder cultivar a través de acuicultura estas especies y comercializarlas con un sistema de trazabilidad adecuado. 


\section{Agradecimientos}

La presente investigación se realizó gracias al apoyo financiero de la Dirección General de Investigación (Digi) de la Universidad de San Carlos de Guatemala (Proyecto No. 4.8.26.2.61), durante el año 2016. Un especial agradecimiento al Conap y a la Dipesca, quienes colaboraron durante el trabajo de campo, así como a los pescadores de Quetzalitos y Livingston por el apoyo brindado.

\section{Referencias}

Alvarado, J. J. (2011). Echinoderm diversity in the Caribbean Sea. Marine Biodiversity, 41, 261-285.

Bordbar, S., Anwar, F., \& Saari, N. (2011). High-value components and bioactives from sea cucumbers for functional food. Marine Drugs, 9(1), 17611805. doi: $10.3390 / \mathrm{md} 9101761$

Borrero Pérez, G. H., Benavides Serrato, M., \& Días Sánchez, C. M. (2012). Equinodermos del Caribe colombiano II: Echinoidea y Holothuroidea. Santa Marta, Colombia: Serie de Publicaciones Especiales de Invemar.

Bruckner, A. W. (Ed.). (2006). Proceedings of the CITES workshop on the conservation of sea cucumbers in the families Holothuridae and Stichopodidae. Silver Spring, United States: National Oceanic and Atmospheric Administration.

Chen, Y., Feindel, S., Kirshenbaum, S., Nutting, G., \& Mercer, L. (2007). An evaluation of the Maine sea cucumber resources and impacts of exploitation. Maine, United States: Northeast Consortium for Cooperative Research.

Consultores Acuícolas y Pesqueros S. C. (2007). Evaluación de las poblaciones silvestres de pepino de mar (Isostichopus fuscus). México: Autor.

Cortés, J. (1997). Comunidades coralinas y arrecifes del Área de Conservación Guanacaste, Costa Rica. Revista de Biología Tropical, 45(1), 623-625.

De la Fuente-Betancourt, M. G., Navarrete, A. J., SosaCordero, E., \& Herrero-Perezrul, M. D. (2001). Assessment of the sea cucumber (Echinodermata: Holothuroidea) as potencial fishery resource in Banco Chinchorro, Quintana Roo, México. Bulletin of marine science, 68(1), 59-67.
De Miras, C., Echeverría, M. A., \& Carranza-Barona, C. (1996). Evaluación socioeconómica de la pesca experimental de pepino de mar en Galápagos. Quito, Ecuador: Fundación Charles Darwin.

Espinoza-Tenorio, A., Pech, D., Ramos, J., \& PeñaPunch, A. (2012). Una radiografía antes de decidir: el reto del aprovechamiento sustentable del pepino de mar en Campeche. Investigación Ambiental, 4(1), 45-50.

Fajardo-León, M. C., Suárez-Higuera, M. C. L., del Valle-Manríquez, A., \& Hernández-López, A. (2008). Reproductive biology of the sea cucumber Parastichopus parvimensis (Echinodermata: Holothuroidea) at Isla Natividad and Bahía Tortugas, Baja California Sur, México. Ciencias Marinas, 34(2), 165-177.

Fonseca, A. C., \& Arrivillaga, A. (2003). Coral reefs of Guatemala: In: J, Cortés. (Ed.). Latin American Coral Reefs. San Pedro, Costa Rica: Elsevier Science.

Fundación Mario Dary Rivera, Consejo Nacional de Áreas Protegidas, \& The Nature Conservancy. (2006). Plan de conservación de área 2007-2011: Refugio De Vida Silvestre Punta De Manabique. Guatemala: Autor.

Guzmán, H. M., \& Guevara, C. A. (2002). Population structure, distribution and abundance of three comercial species of sea cucumber (Echinodermata) in Panama. Caribbean Journal of Science, 38(3-4), 230-238.

Healthy Reefs Initiative. (2015). Mesoamerican Reef: An evaluation of the health of the reef. Florida, United States: Franklin Dodd Communications LLC.

Herrero-Pérezrul, D. (2015). Pepinos de mar: Especies mexicanas sujetas a comercialización y bases para su identificación. México: TRAFFIC/WWF México.

InfoStat. (2008). InfoStat, versión 2008: Manual del usuario. Córdoba, Argentina: Brujas.

López-Rocha, J. A. (2012). Distribución y abundancia del pepino de mar Isostichopus badionotus frente a la Costa de Sisal, Yucatán. Proceedings of the 64th Gulf and Caribbean Fisheries Institute, 1(1), 153-160.

McField, M., Bood, N., Fonseca, A., Arrivillaga, A., Franquesa-Rinos, A., \& Loreto-Viruel, R. M. (s.f.). 
Status of the Mesoamerican Reef after the 2005 Coral Bleaching Event. United States: National Oceanic and Atmospheric Administration.

Ministerio de Agricultura, Ganadería y Alimentación (2010). Acuerdo ministerial 93-2010. Guatemala: Autor.

Poot-Salazar, A., Ardisson, L., Poot Salazar, E., Poot Salazar, D. A., \& Caro Méndez, I. N. (2014). La pesca del pepino de mar en Celestún, Yucatán: Una búsqueda hacia el manejo sostenible. Revista Cubana de Investigaciones Pesqueras, 31(1), 1-4.

Quintanal López, R., Burgos Suarez, L. C., \& Lagunés Vega, J. (2013). El pepino de mar en Yucatán: Una pesca alternativa en desarrollo. Bioagrociencias, 6(2), 39-47.

Rodríguez Gil, L. A., Reyes Sosa, C. F., Alpizar Carrillo, R. A., \& Tello Cetina, J. (2007). Sea cucumber population and biomass estimate for new fishing limit assignation in Sisal fishing cooperative, through the Yucatan State Coast. Proceedings of the 64th Gulf and Caribbean Fisheries Institute, 1(1), 547-553.

Ruíz, J. F., Ibáñez, C. M., \& Cáceres, C. W. (2007). Morfometría del tubo digestivo y alimentación del pepino de mar Athyonidium chilensis (Semper, 1868) (Echinodermata: Holothuroidea). Revista de Biología Marina y Oceanografia, 42(3), 269274.

Ruppert, E. E., \& R. D. Barnes. (1996). Zoología de los invertebrados. México: McGraw-Hill Interamericana.

Salgado-Rogel, M. L., Palleiro-Nayar, J. S., RiveraUlloa, J. L., Aguilar-Montero, D., VásquezSolórzano, E., \& Jiménez-Quiroz, M. del C. (2009). La pesquería y propuestas de manejo del pepino de mar Parastichopus parvimensis en Baja California, México. Ciencia Pesquera, 17(1), 17-26.
Toral, M. V., Martínez, P., Hearn, A., \& Vega, S. (2003). Estado poblacional del pepino de mar (Isostichopus fuscus) en la Reserva Marina de Galápagos: Análisis comparativo de los años 1999-2002. Islas Galápagos, Ecuador: Fundación Charles Darwin.

Toral-Granda, V. (2008). Population status, fisheries and trade of sea cucumbers in Latin America and the Caribbean. In V. Toral-Granda, A. Lovatelli and M. Vasconcellos (Eds.). Sea cucumbers: A global review of fisheries and trade. Roma: Food and Agriculture Organization of the United Nations.

Tuz-Sulub, A., \& Aguilar-Perrera, A. (2011). Aprovechamiento del pepino de mar: pesquería potencial para el desarrollo económico y social en la costa norte de la Península de Yucatán. Bioagrociencias, 4(2), 17-22.

Uthicke, S., \& Benzie, J. A. H. (2001). Restricted gene flow between Holothuria scabra (Echinodermata: Holothuroidea) populations along the northeast coast of Australia and the Solomon Islands. Marine Ecology Progress Series, 216(1), 109117.

Yingst, J. (1982). Factors influencing rates of sediment ingestión by Parastichopus parvimensis (Clark), an epibenthic deposit feeding Holothurian. Estuarine Coastal and Shelf Science 141(1), 119-134 